\title{
Rigidity and Persistence of Meta-Formations
}

\author{
Julien M. Hendrickx, Changbin Yu, Barış Fidan and Brian D.O. Anderson
}

\begin{abstract}
This paper treats the problem of the merging of formations, where the underlying model of a formation is graphical. We first analyze the persistence of meta-formations, which are formations obtained by connecting several persistent formations. Persistence is a generalization to directed graphs of the undirected notion of rigidity. In the context of moving autonomous agent formations, persistence characterizes the efficacy of a directed structure of unilateral distance constraints seeking to preserve a formation shape. We derive then, for agents evolving in a two- or three-dimensional space, the conditions under which a set of persistent formations can be merged into a persistent meta-formation, and give the minimal number of interconnections needed for such a merging. We also give conditions for a meta-formation obtained by merging several persistent formations to be persistent.
\end{abstract}

\section{INTRODUCTION}

By autonomous agent, we mean here any humancontrolled or unmanned vehicle moving by itself and having a local intelligence or computing capacity, such as ground robots, air vehicles or underwater vehicles. Significant interest has been shown on the behavior of autonomous agent formations (groups of autonomous agents interacting which each other) [2]-[5], [10], and more recently on meta-formations, consisting of interconnected formations [1], [14]. Many reasons such as obstacle avoidance and dealing with a predator can indeed lead a (meta-)formation to be split into smaller formations which are later re-merged. Those smaller formations need to be organized in such a way that they can behave autonomously when the formation is split. Conversely, some formations may need to be temporarily merged into a meta-formation to accomplish a certain task, this meta-formation being split afterwards.

The particular property of formations and meta-formations which we analyze here is persistence. This graph-theoretical

J. M. Hendrickx is with Department of Mathematical Engineering, Université catholique de Louvain, Avenue Georges Lemaitre 4, B-1348 Louvain-la-Neuve, Belgium; hendrickx@inma.ucl.ac.be. His work is supported by the Belgian Programme on Interuniversity Attraction Poles initiated by the Belgian Federal Science Policy Office, and the Concerted Research Action (ARC) "Large Graphs and Networks" of the French Community of Belgium. The scientific responsibility rests with its authors. Julien Hendrickx holds a FNRS fellowship (Belgian Fund for Scientific Research)

C. Yu, B. Fidan and B. Anderson are with Australian National University and National ICT Australia, 216 Northbourne Ave, Canberra ACT 2601 Australia ; brad.yu, baris.fidan, brian.anderson@nicta.com.au. Their work is supported by an Australian Research Council Discovery Project Grant and by National ICT Australia, which is funded by the Australian Government's Department of Communications, Information Technology and the Arts and the Australian Research Council through the Backing Australia's Ability Initiative. Changbin Yu is an AustraliaAsia Scholar supported by the Australian Government's Department of Education, Science and Training through Endeavours Programs. notion was introduced in [5] to analyze the behavior of autonomous agent formations governed by unilateral distance constraints: Many applications require some interagent distances to be kept constant during a continuous move in order to preserve the shape of a multi-agent formation. In other words, when enough inter-agent distances are explicitly maintained constant, all the inter-agent distances remain constant. The information structure arising from such a system can be efficiently modelled by a graph, where agents are abstracted by vertices and actively constrained inter-agent distances by edges. We assume here that those constraints are unilateral, i.e., that the responsibility for maintaining a distance is not shared by the two concerned agents but relies on only one of them. This can be a deliberate choice to improve the efficacy or the stability of the formation, but also a consequence of some technical constraints: Some UAV's can for instance not sense the objects located behind them. This asymmetry is modelled using directed edges in the graph. Intuitively, an information structure is persistent if, provided that each agent is trying to satisfy all the distance constraints for which it is responsible, all the inter-agent distances remain constant and as a result the formation shape is preserved. A necessary but not sufficient condition for persistence is rigidity, which intuitively means that, provided that all the prescribed distance constraints are satisfied during a continuous displacement, all the inter-agent distances remain constant. The above notion of rigidity can also be applied to structural frameworks where the vertices correspond to joints and the edges to bars. The main difference between rigidity and persistence is that rigidity assumes all the constraints to be satisfied, as if they were enforced by an external agency or through some mechanical properties, while persistence considers each constraint to be the responsibility of a single agent. As explained in [5], persistence implies rigidity, but it also implies that the responsibilities imposed on each agent are not inconsistent, for there can indeed be situations where this is so, and they must be avoided. Rigidity is thus an undirected notion (not depending on the edge directions), while persistence is a directed one. Both rigidity and persistence can be analyzed from a graph-theoretical point of view, and it can be proved [5], [12], [17] that if a formation is rigid (resp. persistent), then almost all formations represented by the same graph are rigid (resp. persistent).

As stated in [1], the problem of merging rigid formations into a rigid meta-formation has been considered in a number of places. In [9], [11], the rigidity of a multi-graph (a graph 


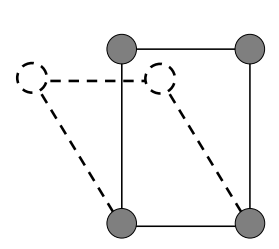

(a)

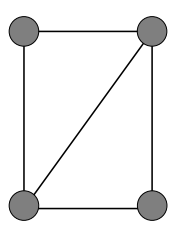

(b)

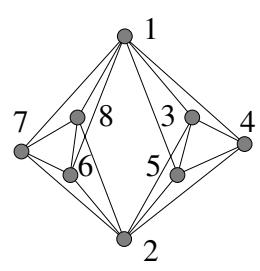

(c)
Fig. 1. In $\Re^{2}$, the graph represented in (a) is not rigid because it can be deformed (dashed line), while the one in (b) is rigid. The graph (c) satisfies the $3 \mathrm{D}$ condition analogous to Theorem 1 but is not rigid in $\Re^{3}$ : the two parts of the graph can rotate around the axis defined by 1 and 2 .

in which some vertices are abstractions of smaller graphs) is analyzed. The vertices of a multi-graph can be thought as two dimensional solid bodies at the surface of which some bars can be attached; two vertices are then connected by an edge if the corresponding bodies are attached to a same bar. Operational ways to merge two rigid formations into a larger rigid formation can also be found in [4], [15].

In this paper, we treat the problem of determining whether a given meta-formation obtained by merging several persistent formations is persistent. For this purpose, we first consider the above mentioned problem of determining whether a meta-formation obtained by merging rigid formations is rigid. We also analyze the conditions under which a collection of persistent formations can be merged into a persistent meta-formation. Conditions are then given on the minimal number of additional links that are needed to achieve such a merging. Note that throughout all the paper, we always assume that the internal structure of the formations cannot be modified. Moreover, we use a convenient graph theoretical formalism, abstracting agents by vertices and (unilateral) distance constraints by (directed) edges.

After reviewing some properties of rigidity and persistence of graphs in Section II, we examine in Section III the issues mentioned above for agents evolving in a two-dimensional space. We show in Section IV how our results can be generalized in a three-dimensional space, and explain why this generalization can only partially be achieved. The paper ends then by the concluding remarks in Section V. Due to space limitations the proofs of some results are omitted, but they are available on request from the authors.

\section{Review of Rigidity And Persistence}

As explained in Section I, the rigidity of a graph has the following intuitive meaning: Suppose that each vertex represents an agent in a formation, and each edge represents an inter-agent distance constraint enforced by an external observer. The graph is rigid if for almost every such structure, the only possible continuous moves are those which preserve every inter-agent distance, as shown in Fig. 1(a) and (b). For a more formal definition, the reader is referred to [5], [12]. In $\Re^{2}$, that is, if the agents represented by the vertices of the

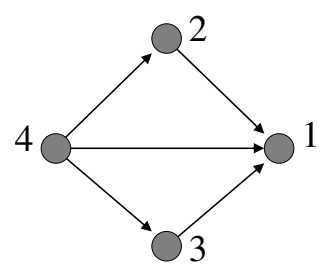

(a)

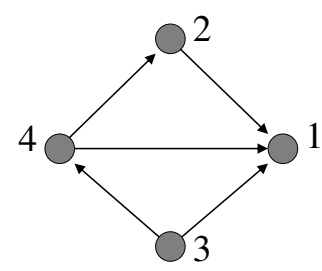

(b)
Fig. 2. In $\Re^{2}$, the graph represented in (a) is rigid but not persistent. For almost all uncoordinated displacements of 2, 3 and 4 (even if they satisfy their constraints), 4 is indeed unable to satisfy its three constraints. This problem cannot happen for the graph represented in (b), which is persistent.

graph evolve in two dimensions, there exists a combinatorial criterion to check if a given graph is rigid:

Theorem 1 (Laman [8], [13]): A graph $G=(V, E)$, with $|V|>1$, is rigid in $\Re^{2}$ if and only if there is a sub-set $E^{\prime} \subseteq E$ such that

- $\left|E^{\prime}\right|=2|V|-3$.

- For all non-empty $E^{\prime \prime} \subseteq E^{\prime}$ there holds $\left|E^{\prime \prime}\right| \leq 2\left|V\left(E^{\prime \prime}\right)\right|-3$, where $V\left(E^{\prime \prime}\right)$ is the set of vertices incident to edges of $E^{\prime \prime}$.

Unfortunately the analogous criterion in $\Re^{3}$ obtained by replacing respectively 2 by 3 and 3 by 6 is only necessary, as demonstrated by the example in Fig. 1(c). We say that a graph is minimally rigid if it is rigid and if no single edge can be removed without losing rigidity. It follows from the results above that a graph is minimally rigid in $\Re^{2}$ (resp. in $\Re^{3}$ ) if and only if it is rigid and contains $2|V|-3$ (resp. $3|V|-6)$ edges [12].

Consider now that the constraints are not enforced by an external entity, but that each constraint is the responsibility of one agent to enforce. To each agent, one assigns a (possibly empty) set of unilateral distance constraints represented by directed edges: the notation $(i, j)$ for a directed edge connotes that the agent $i$ has to maintain its distance to $j$ constant during any continuous move. As explained in the Introduction, the persistence of the directed graph means that provided that each agent is trying to satisfy its constraints, the distance between any pair of connected or non-connected agents is maintained constant during any continuous move, and as a consequence the shape of the formation is preserved. Note though that the assignments given to an agent may be impossible to fulfill, in which case persistence is not achieved. An example of a persistent and a non-persistent graph having the same underlying undirected graph is shown in Fig. 2. For a more formal definition of persistence, the reader is referred to [5], [17], where it is also proved that a persistent graph is always rigid, and that persistence can be checked by checking the rigidity of several subgraphs. A key result in the proof of this is the following:

Proposition 1: A persistent graph $\Re^{2}$ (resp. $\Re^{3}$ ) remains persistent after removal of an edge leaving a vertex whose out-degree is larger than 2 (resp. 3). 
We use the term number of degrees of freedom of a vertex $i$ to denote the (generic) dimension of the set in which the corresponding agent can choose its position (all the other agents being fixed). Thus it represents in some sense the decision power of this agent. The number of degrees of freedom of a vertex $i$ in $\Re^{2}$ (resp. $\Re^{3}$ ) is given by $\max \left(0,2-d^{+}(i)\right)\left(\right.$ resp. $\left.\max \left(0,2-d^{+}(i)\right)\right)$, where $d^{+}(i)$ represent the out-degree of the vertex $i$. A vertex having a maximal number of degrees of freedom (i.e. an out-degree 0 ) is called a leader since the corresponding agent does not have any distance constraint to satisfy. It is proved in [5], [17] that the sum of the numbers of degrees of freedom over all vertices of a persistent graph cannot exceed 3 in $\Re^{2}$ and 6 in $\Re^{3}$. Note that those numbers correspond to the number of independent translations and rotations in $\Re^{2}$ and $\Re^{3}$. In the sequel we abbreviate degree of freedom by DOF. By an abuse of language, we define the DOF number of a graph to be the sum of the DOF numbers of degrees of freedom of its vertices.

As explained in [17], although the concept of persistence is applicable in three and larger dimensions, it is not sufficient to imply the desired stability of the formation shape. For the shape stability, the graph corresponding to a three-dimensional formation needs indeed to be structurally persistent. In $\Re^{3}$, a graph is structurally persistent if and only if it is persistent and contains at most one leader. In $\Re^{2}$, persistence and structural persistence are equivalent.

Similarly to minimal rigidity, we say that a graph is minimally (structurally) persistent if it is (structurally) persistent and if no single edge can be removed without losing (structural) persistence. It is proved in [5], [17] that a graph is minimally (structurally) persistent if and only if it is (structurally) persistent and minimally rigid. The number of edges of such a graph is thus uniquely determined by the number of its vertices as it is the case for minimally rigid graphs.

\section{Rigidity AND PERSistence OF 2D META-FORMATIONS}

\section{A. Rigidity}

Consider a set $N$ of disjoint rigid (in $\Re^{2}$ ) graphs $G_{1}, \ldots, G_{|N|}$ having at least two vertices, and a set $S$ of single-vertex graphs $G_{|N|+1}, \ldots, G_{|N|+|S|}$. In the sequel, those graphs are called meta-vertices, and it is assumed that no modification can be made on their internal structure: no internal edge or vertex can be added to or removed from a meta-vertex. We define the merged graph $G$ by taking the union of all the meta-vertices, and of some additional edges $E_{M}$ each of which has end-points belonging to different meta-vertices.

The conditions under which the merging of two metavertices leads to a rigid graph are detailed in [15]: If both meta-vertices contain more than one vertices, the merged graph is rigid if and only if $E_{M}$ contains at least three edges,

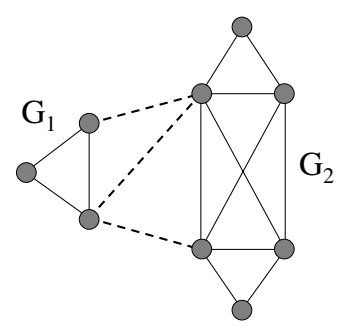

(a)

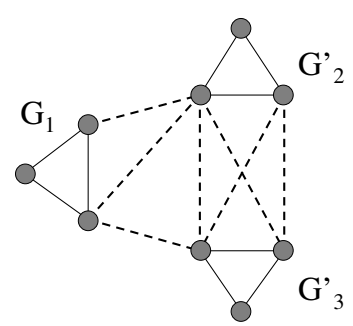

(b)
Fig. 3. The graph represented in (a) and (b) is an edge-optimal rigid merge if it is obtained by merging $G_{1}$ and $G_{2}$ (a) but not if it is obtained by merging $G_{1}, G_{2}^{\prime}$ and $G_{3}^{\prime}$ (c). The dashed edge represents the edges of $E_{M}$

which are incident to at least two vertices of each metavertex. This is actually a particular case of the following result for an arbitrary number of graphs (analogous to a result in [9] which is obtained under the assumption that no vertex of any meta-vertex is incident to more than one edge of $\left.E_{M}\right)$ :

Theorem 2: $G=\left(\cup_{N, S} G_{i}\right) \cup E_{M}$ (with $N$ and $S$ as defined at the beginning of this section) is rigid if and only if there exists $E_{M}^{\prime} \subseteq E_{M}$ such that

- $\left|E_{M}^{\prime}\right|=3|N|+2|S|-3$

- For all non-empty $E_{M}^{\prime \prime} \subseteq E_{M}^{\prime}$, there holds $\left|E_{M}^{\prime \prime}\right| \leq 3\left|I\left(E_{M}^{\prime \prime}\right)\right|+2\left|J\left(E_{M}\right)\right|-3$

where $I\left(E_{M}^{\prime \prime}\right)$ is the set of meta-vertices with at least two vertices incident to edges of $E_{M}^{\prime \prime}$ and $J\left(E_{M}^{\prime}\right)$ is the set of those with only one vertex incident to edge(s) of $E_{M}^{\prime \prime}$.

For a given collection of meta-vertices, we say that $G$ is an edge-optimal rigid merging if no single edge of $E_{M}$ can be removed without losing rigidity. Notice that a single graph can be an edge-optimal rigid merging with respect to a certain collection of meta-vertices, and not with respect to another one, as shown in Fig. 3. If all meta-vertices are minimally rigid, then an edge-optimal rigid merging is also a minimally rigid graph. From Theorem 2, one can deduce that $G$ is an edge-optimal rigid merging if and only if it is rigid and $\left|E_{M}\right|=3|N|+2|S|-3$.

\section{B. Persistence}

Next we analyze the case where the meta-vertices are directed persistent graphs. If it is possible to merge them into a persistent graph, then it is possible to do so in such a way that all the edges of $E_{M}$ leave vertices which have an out-degree not greater than 2 in $G$. A set of edges $E_{M}$ that would make $G$ persistent but that would not satisfy this property could indeed be reduced by Proposition 1 until it satisfies it. It is possible to prove that when the added edges leave only vertices whose out-degree is not greater than 2 , $G$ is persistent if and only if it is rigid. The condition on the out-degrees of the vertices with an outgoing edge of $E_{M}$ can be conveniently re-expressed in terms of degrees of freedom: To each DOF (within a single meta-vertex) of any vertex there corresponds at most one outgoing edge of $E_{M}$. By an abuse of language, we say that such edges leave a vertex with one or more local DOFs, i.e. a vertex which 


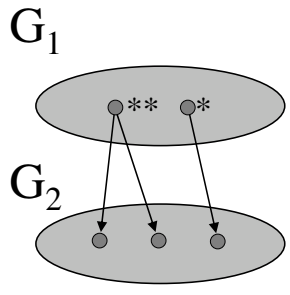

(a)

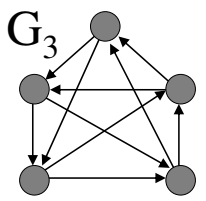

(b)

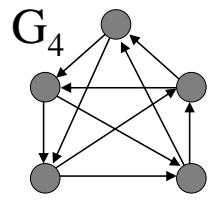

Fig. 4. Merging of the persistent meta-vertices $G_{1}$ and $G_{2}$ into a persistent graph in $\Re^{2}$ (a). The symbol "*" represents one DOF (with respect to the meta-vertex). (b) represents two persistent meta-vertex that cannot be merged into a persistent graph in $\Re^{2}$ because none of their vertices has a DOF.

inside its meta-vertex has one or more DOFS. This allows reformulating the above results in a dimension-free way:

Theorem 3: A collection of (structurally) persistent meta-vertices can be merged into a (structurally) persistent graph if and only if it can be merged into a (structurally) persistent graph by adding edges leaving vertices with one or more local DOFs. In that case, the merged graph is persistent if and only if it is rigid.

If one or more edges do leave a vertex with an out-degree larger than 2, no criterion has been found yet to determine whether the merged graph is persistent or not, which also takes advantage of the fact that the graph is obtained by merging several persistent meta-vertices.

Tying Theorem 3 together with what is known and reviewed above regarding the merging of two rigid metavertices, we conclude: two persistent meta-vertices $G_{a}$ and $G_{b}$ each having more than one vertex can be merged into a persistent graph if and only if three edges leaving vertices with local DOFs can be added in such a way that they are incident to at least two vertices in each meta-vertex. There must thus be at least three local DOFs available among the vertices in $G_{a}$ and $G_{b}$. Conversely, if there are available three local DOFs among the vertices of $G_{a}$ and $G_{b}$, since no vertex can have more than two DOFs, it is possible to add a total of at least three edges leaving at least two vertices of $G_{a} \cup G_{b}$. The vertices to which those edges arrive can then be chosen in such a way that at least two vertices of both $G_{a}$ and $G_{b}$ are incident to edges of $E_{M}$, as shown in Fig. 4. By Theorem 3, this implies that the merged graph would be rigid and therefore persistent:

Proposition 2: Two meta-vertices with more than one vertex can be merged into a persistent graph if and only if the sum of their DOFs number is at least 3. At least three edges are needed to perform this merging, and merging can always be done with exactly three edges.

If one or two of the meta-vertices are single vertex graphs, the result still holds, but the minimal number of added edges (and therefore the number of needed DOFs) are then respectively 2 and 1 . We define the number of missing DOFs $\left(m_{D O F}\right)$ to be the absolute value of the difference between the DOF number of the graph and the maximal DOF number that any graph with the same number of vertices can have. In $\Re^{2}$, this maximal number is 2 for the single vertex graphs, and 3 for other persistent graphs. There is an interesting consequence: when the minimal number of edges is used to merge two meta-vertices $G_{a}$ and $G_{b}$, the number of missing DOFs is preserved through the process, i.e. $m_{D O F}\left(G_{a} \cup G_{b} \cup E_{M}\right)=m_{D O F}\left(G_{a}\right)+m_{D O F}\left(G_{b}\right)$.

Consider now an arbitrary number of meta-vertices, possibly containing single-vertex graphs, but such that the total number of vertices is at least 2 . If the sum of their number of missing DOFs is no greater than 3, it follows from Proposition 2 that any two of them can be merged in such a way that the obtained graph is persistent and that the total number of missing DOFs remains unchanged. Any pair of those meta-vertices would indeed contain at least the required number of DOFs. Doing this recursively, it is possible to merge all these meta-vertices into a single persistent graph. In case there are more than 3 missing DOFs, the total DOF number is by definition smaller than $3|N|+2|S|-3$, which is the minimal number of edges required to make the merged graph rigid. It follows then from Theorem 3 that such metavertices cannot be merged in a persistent graph. We have thus proved the following result:

Proposition 3: A collection of persistent meta-vertices $N \cup S$ (with $N$ and $S$ as defined in the beginning of Section III-A) can be merged into a persistent graph if and only if the total number of missing DOFs is no greater than 3 , or equivalently if the total number of local DOF in $N \cup S$ is at least $3|N|+2|S|-3$. At least $3|N|+2|S|-3$ edges are needed to perform this merging, and merging can always be done with exactly this number of edges.

As when merging rigid meta-vertices, we say that $G$ is an edge-optimal persistent merging if no single edge of $E_{M}$ can be removed without losing persistence. Again, if all metavertices are minimally persistent, then $G$ is an edge-optimal persistent merging if and only if it is minimally persistent. It can be proved that:

Theorem 4: $G=\left(\bigcup_{N, S} G_{i}\right) \cup E_{M}$ (with $N$ and $S$ as defined at the beginning of Section III-A and with all $G_{i}$ persistent) is an edge-optimal persistent merging in $\Re^{2}$ if and only if the following conditions all hold

- $\left|E_{M}\right|=3|N|+2|S|-3$

- For all non-empty $E_{M}^{\prime \prime} \subseteq E_{M}^{\prime}$, there holds $\left|E_{M}^{\prime \prime}\right| \leq 3\left|I\left(E_{M}^{\prime \prime}\right)\right|+2\left|J\left(E_{M}\right)\right|-3$ with $I\left(E_{M}^{\prime \prime}\right)$ and $J\left(E_{M}^{\prime}\right)$ as defined in Theorem 2

- All edges of $E_{M}$ leave vertices with local DOFs.

Equivalently, a persistent $G$ is an edge-optimal persistent merging if and only if it is an edge-optimal rigid merging, or if and only if its number of missing DOFs is equal to the sum of the numbers of missing DOFs of all its metavertices. Notice that an efficient way to obtain such a merging is provided in the discussion of Proposition 3. 


\begin{tabular}{c|rrrrrr}
$\left|V_{a}\right|$ & 1 & 1 & 1 & 2 & 2 & $\geq 3$ \\
\hline$\left|V_{b}\right|$ & 1 & 2 & $\geq 3$ & 2 & $\geq 3$ & $\geq 3$ \\
\hline $\min \left|E_{M}\right|$ & 1 & 2 & 3 & 3 & 5 & 6
\end{tabular}

TABLE I

MIN. NUMBER OF EDGES TO MERGE TWO GRAPH INTO A RIGID GRAPH.

\section{Rigidity AND PeRsistence of 3D META-FORMATIONS}

\section{A. Rigidity}

We now consider a set $N$ of disjoint rigid (in $\Re^{3}$ ) graphs $G_{1}, \ldots, G_{|N|}$ having at least three vertices, a set $D$ of graphs containing two (connected) vertices $G_{|N|+1}, \ldots, G_{|N|+|D|}$, and a set $S$ of single-vertex graphs $G_{|N|+|D|+1}, \ldots, G_{|N|+|D|+|S|}$. As in Section III, these graphs are called meta-vertices, and we define the merged graph $G$ by taking the union of all the meta-vertices, and of some additional edges $E_{M}$ each of which having end-points belonging to different meta-vertices.

The merging of two rigid meta-vertices, each containing more than two vertices, is treated in [15]: At least six edges are needed, and they must be incident to at least three vertices of each meta-vertex, and it is always possible to achieve a rigid merging using exactly six edges incident to exactly three vertices of each meta-vertex. But these conditions are only necessary: The so-called "double-banana" of Fig. 1(c) can be obtained by merging two distinct rigid tetrahedral meta-vertices $(1,3,4,5)$ and $(2,5,7,8)$ using a total of six edges incident to four vertices of each meta-vertex. With a minor modification, the merging result above holds in the cases where at least one meta-vertex has less than 3 vertices: The required number of edges is different, as summarized in Table I where $\min \left|E_{M}\right|$ represents the minimal number of edges required to merge the meta-vertices $G_{a}\left(V_{a}, E_{a}\right)$ and $G_{b}\left(V_{b}, E_{b}\right)$ into a rigid graph. Also, if a meta-vertex has less than 3 vertices, all of them should be incident to edges of $E_{M}$, otherwise at least 3 of them should be. When merging several meta-vertices, there is no available necessary and sufficient condition for the rigidity of $G$. Determining whether a merged graph is rigid in $\Re^{3}$ is indeed a more general problem than determining whether a given graph is rigid (it suffices to take $N=D=\varnothing$ ) and there is no known set of combinatorial necessary and sufficient conditions for this. However, we have the following necessary condition:

Theorem 5: $G=\left(\bigcup_{N, D, S} G_{i}\right) \cup E_{M}$ (whith $N, S, D$ as defined at the beginning of this section) is rigid (in $\Re^{3}$ ). Then there exists $E_{M}^{\prime} \subseteq E_{M}$ such that

- $\left|E_{M}^{\prime}\right|=6|N|+5|D|+3|S|-6$

- for all non-empty $E_{M}^{\prime \prime} \subseteq E_{M}^{\prime}$, there holds $\left|E_{M}^{\prime \prime}\right| \leq 6\left|I\left(E_{M}^{\prime \prime}\right)\right|+5\left|J\left(E_{M}^{\prime \prime}\right)\right|+3\left|K\left(E_{M}^{\prime \prime}\right)\right|-6$,

where $I\left(E_{M}^{\prime \prime}\right)$ is the set of meta-vertices with at least three vertices or two unconnected ones incident to edges of $E_{M}^{\prime \prime}$, $J\left(E_{M}^{\prime \prime}\right)$ is the set of those with one connected pair incident to edges of $E_{M}^{\prime \prime}$, and $K\left(E_{M}^{\prime \prime}\right)$ the set of those with only

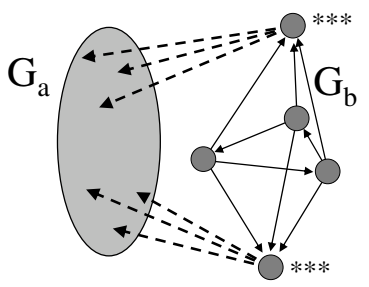

(a)

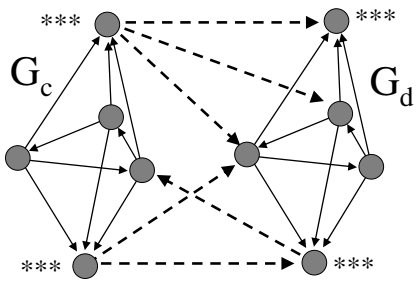

(b)
Fig. 5. Example of a persistent but not structurally persistent meta-vertex $G_{b}$ which cannot be merged into a persistent or rigid graph with the metavertex $G_{a}$, the latter being persistent but having no DOF. (b) shows how two non-structurally persistent meta-vertices can be merged into a structurally persistent graph. The symbol "**" represents one DOF, and the dashed edges are the edges of $E_{M}$.

one vertex incident to edge(s) of $E_{M}^{\prime \prime}$.

It should be noted that the counting conditions of Theorem 5 are not sufficient for rigidity. The non-rigid graph of Fig. 1 (c) which can be obtained by merging two rigid tetrahedral meta-vertices $(1,3,4,5)$ and $(2,6,7,8)$ would indeed satisfy them. Nevertheless, one can deduce from Theorem 5 that $G$ is an edge-optimal rigid merging in $\Re^{3}$ if and only if it is rigid and $\left|E_{M}\right|=6|N|+5|D|+3|S|-6$.

\section{B. Persistence}

Consider now all meta-vertices as persistent graphs. Theorem 3 can be proved in a way that does not depend on the dimension of the space in which the agent evolves and is thus still valid in three dimensions. Merging two meta-vertices into a persistent graph is however a more complicated problem in $\Re^{3}$ than in $\Re^{2}$. Consider indeed a meta-vertex $G_{a}$ without any DOF, and a meta-vertex $G_{b}$ which is not structurally persistent, i.e. which is persistent and contains two vertices (leaders) having three DOFs. The number of available DOFs is equal to the minimal number of edges that should be added to obtain a rigid merged graph. However, the only way to add six edges leaving local DOFs is to add three edges leaving each leader of $G_{b}$ and arriving in $G_{a}$, as shown in Fig. 5(a). Only two vertices of $G_{b}$ would thus be incident to the added edges, which prevents the merged graph from being rigid and therefore persistent. We have thus proved the following condition:

Proposition 4: If two persistent meta-vertices are such that one is not structurally persistent and the other does not have any DOF, they cannot be merged into a persistent graph.

However, this is the only case for which the argument used in establishing Proposition 2 cannot be generalized to establish an analogous property in $\Re^{3}$ :

Proposition 5: If two meta-vertices (with more than 2 vertices) do not satisfy the condition of Proposition 4, they can be merged into a persistent graph if and only if the sum of their DOFs is at least 6. At least six edges are needed to perform this merging, and merging can always be done with exactly six edges and in such a way that the graph obtained is structurally persistent. 
In case at least one of the meta-vertices has less than 3 vertices, the result still holds, but with a different required number of edges in $E_{M}$ and therefore of available DOFs: these minimal numbers are both equal to $\min \left|E_{M}\right|$ in Table I (for the merging of a graph $G_{a}\left(V_{a}, E_{a}\right)$ with a graph $G_{b}\left(V_{b}, E_{b}\right)$ ). It is worth noting that even if one or both of the meta-vertices are not structurally persistent, it is possible to obtain a structurally persistent merged graph, as shown in Fig. 5(b). This has already been observed in [17] for the case where one meta-vertex is a single vertex graph.

Consider now a collection of meta-vertices such that the total number of vertices is at least 3. By a similar argument as for Proposition 3, one can prove the following result.

Proposition 6: A collection of persistent meta-vertices $N \cup D \cup S$ (with $N, D, S$ as defined in the beginning of Section IV-A) that does not consist of only two meta-vertices satisfying the condition of Proposition 4 can be merged into a persistent graph if and only if the total number of missing DOFs is no greater than 6, or equivalently if the total number of local DOFs in $N \cup D \cup S$ is at least $6|N|+5|D|+3|S|-6$. At least $6|N|+5|D|+3|S|-6$ edges are needed to perform this merging. Merging can always be done with exactly this number of edges, and in such a way that the merged graph is structurally persistent.

The only difference with the discussion of Proposition 3 appears when the collection contains two meta-vertices satisfying the condition of Proposition 4 (that is, one not structurally persistent and one without DOF) and at least one other meta-vertex. In that case, a counting argument shows that there can only be one meta-vertex with no DOF, and that apart from this one, all meta-vertices have no missing DOF. One can thus begin by merging all those into a structurally persistent graph, which can then be merged with the meta-vertex without DOF.

As in the two-dimensional case, one can prove that a persistent graph is an edge-optimal persistent merging if and only if it is an edge-optimal rigid merging. However, due to the absence of necessary and sufficient conditions allowing a combinatorial checking of the rigidity of a graph or of a merged graph in $\Re^{3}$, the result cannot be expressed in a purely combinatorial way.

Theorem 6: $G=\left(\bigcup_{N, D, S} G_{i}\right) \cup E_{M}$ with $N, D, S$ as defined at the beginning of Section IV-A and with all $G_{i}$ persistent) is an edge-optimal persistent merging in $\Re^{3}$ if and only if the following conditions all hold

- $\left|E_{M}\right|=6|N|+5|D|+3|S|-6$

- $G$ is rigid

- All edges of $E_{M}$ leave local DOFs.

Again, an efficient way to obtain an edge-optimal persistent merging from a collection of meta-vertices satisfying the hypotheses of Proposition 6 is to first merge two of them and then to iterate, as in the discussion of Propositions 3 and 6.

\section{Conclusions}

We have analyzed the conditions under which a graph resulting from the merging of several persistent graphs is itself persistent. Necessary and sufficient conditions were found to determine which collections of persistent graphs could be merged into a larger persistent graph. We first treated these issues in $\Re^{2}$. Our analysis was then generalized to $\Re^{3}$ and to structural persistence, leading to somewhat less powerful results. This is especially the case for those which rely on the sufficient character of Laman's conditions for rigidity in $\Re^{2}$ (Theorem 1), no equivalent condition being known in $\Re^{3}$. Following this work, we plan to develop systematic ways to build all optimally merged persistent graphs, similarly to what has been done for minimally persistent graphs [6] and for minimally rigid merged graphs [16].

\section{REFERENCES}

[1] B.D.O. Anderson, C. Yu, B. Fidan, and J.M. Hendrickx. Use of meta-formations for cooperative control. Proc. of the 17th International Symposium on Mathematical Theory of Networks and Systems (MTNS2006), pages 2381-2387 Kyoto (Japan), July $2006 .$.

[2] J. Baillieul and A. Suri. Information patterns and hedging Brockett's theorem in controlling vehicle formations. Proc. of the 42nd IEEE Conf. on Decision and Control, volume 1, pages 556-563, Hawaii (HA), USA, December 2003.

[3] A. Das, R. Fierro, V. Kumar, and Ostrowski J.P. A vison based formation control framework. IEEE trans. on Robotics and Automation, 18(5):813-825.

[4] T. Eren, B.D.O. Anderson, W. Whiteley, A.S. Morse, and P.N. Belhumeur. Operations on rigid formations of autonomous agents. Communications in Onformations and Systems, 3(4):223-258, 2004.

[5] J.M. Hendrickx, B.D.O. Anderson, J.-C. Delvenne, and V.D. Blondel. Directed graphs for the analysis of rigidity and persistence in autonomous agents systems. To appear in International Journal of Robust and Nonlinear Control's special issue on CommunicatingAgent Systems.

[6] J.M. Hendrickx, B. Fidan, C. Yu, B.D.O. Anderson, and V.D. Blondel. Primitive operations for the construction and reorganization of minimally persistent formations. Submitted for publication.

[7] D.J. Jacobs and B. Hendrickson. An algorithm for two-dimensional rigidity percolation: the pebble game. J. Comput. Phys., 137(2):346365, 1997.

[8] G. Laman. On graphs and rigidity of plane skeletal structures. J. Engrg. Math., 4:331-340, 1970.

[9] C. Moukarzel. An efficient algorithm for testing the generic rigidity of graphs in the plane. J. Phys. A, 29(24):8079-8098, 1996.

[10] R. Olfati-Saber and R.M Murray. Graph rigidity and distributed formation stabilization of multi-vehicle systems. Proc. of the 41st Conference on Decision and Control, Las Vegas (NV), USA, December 2002 .

[11] T.S. Tay. Rigidity of multigraphs. I. Linking rigid bodies in $n$-space. J. Combin. Theory Ser. B, 36(1):95-112, 1984.

[12] T.S. Tay and W. Whiteley. Generating isostatic frameworks. Structural Topology, (11):21-69, 1985.

[13] W. Whiteley. Some matroids from discrete applied geometry. Matroid theory (Seattle, WA, 1995), volume 197 of Contemp. Math., pages 171-311. Amer. Math. Soc., Providence, RI, 1996.

[14] A. Williams, S. Glavaški, and T. Samad. Formations of formations: Hierarchy and stability. Proc. of the 2004 American Control Conference, pages 2992-2997, Boston (MA), USA, July 2004.

[15] C. Yu, B. Fidan, and B.D.O. Anderson. Principles to control autonoumous formation merging. Proc. of the 2006 American Control Conference, pages 762-768, Minneapolis (MN), USA, June 2006.

[16] C. Yu, B. Fidan, J.M. Hendrickx, and B.D.O. Anderson. Merging multiple formations: a meta-formation prospective. To appear in the Proc. of the 45st Conference on Decision and Control, San Diego (CA), USA, December 2006.

[17] C. Yu, J.M. Hendrickx, B. Fidan, B.D.O. Anderson, and V.D. Blondel. Three and higher dimensional autonomous formations: Rigidity, persistence and structural persistence. To appear in Automatica 2007. 